\title{
Performance and Portability of Accelerated Lattice Boltzmann Applications with OpenACC
}

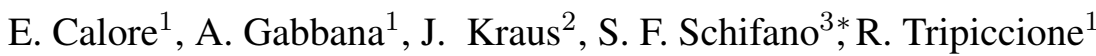 \\ ${ }^{1}$ Dip. di Fisica e Scienze della Terra, University of Ferrara, and INFN, Ferrara (Italy) \\ ${ }^{2}$ NVIDIA GmbH, Würselen (Germany) \\ ${ }^{3}$ Dip. di Matematica e Informatica, University of Ferrara, and INFN, Ferrara (Italy)
}

\begin{abstract}
SUMMARY
An increasingly large number of HPC systems rely on heterogeneous architectures combining traditional multi-core CPUs with power efficient accelerators. Designing efficient applications for these systems has been troublesome in the past as accelerators could usually be programmed using specific programming languages threatening maintainability, portability and correctness. Several new programming environments try to tackle this problem. Among them, OpenACC offers a high-level approach based on compiler directives to mark regions of existing $\mathrm{C}, \mathrm{C}++$ or Fortran codes to run on accelerators. This approach directly addresses code portability, leaving to compilers the support of each different accelerator, but one has to carefully assess the relative costs of portable approaches versus computing efficiency. In this paper we address precisely this issue, using as a test-bench a massively parallel Lattice Boltzmann algorithm. We first describe our multinode implementation and optimization of the algorithm, using OpenACC and MPI. We then benchmark the code on a variety of processors, including traditional CPUs and GPUs, and make accurate performance comparisons with other GPU implementations of the same algorithm using CUDA and OpenCL. We also asses the performance impact associated to portable programming, and the actual portability and performance-portability of OpenACC-based applications across several state-of-the-art architectures.

This is the pre-peer reviewed version of the following article: Performance and Portability of Accelerated Lattice Boltzmann Applications with OpenACC. (2016) Concurrency Computat.: Pract. Exper., 28: 3485-3502, which has been published in final form at 10.1002/cpe.3862. This article may be used for non-commercial purposes in accordance with Wiley Terms and Conditions for Self-Archiving. Copyright (C) 0000 John Wiley \& Sons, Ltd.
\end{abstract}

Received ...

KEY WORDS: OpenACC, Lattice Boltzmann Methods, Performance Analysis

\section{INTRODUCTION, RELATED WORKS AND BACKGROUND}

Lattice Boltzmann (LB) methods are widely used in computational fluid dynamics, to simulate flows in two and three dimensions. From the computational point of view, LB methods have a large degree of available parallelism so they are suitable for massively parallel systems.

Over the years, LB codes have been written and optimized for large clusters of commodity CPUs [1], for application-specific machines [2, 3, 4] and even for FPGAs [5]. More recently work has focused on exploiting the parallelism of powerful traditional many-core processors [6], and of power-efficient accelerators such as GPUs [7, 8] or Xeon-Phi processors [9].

As diversified HPC architectures emerge, it is becoming more and more important to have robust methodologies to port and maintain codes for several architectures. This need has sparked the development of frameworks, such as the Open Computing Language (OpenCL), allowing to write portable codes, that can be compiled (with varying degrees of efficiency) for several accelerator architectures. OpenCL is a low level approach: it usually obtains high performances at the price of 
substantial adjustments in the code implying large human efforts and seriously posing a threat to code correctness and maintainability.

Other approaches start to emerge, mainly based on directives: compilers generate offloadfunctions for accelerators, following "hints" provided by programmers as annotations to the original - C, C++ or Fortran - codes [10]. Examples along this direction are OpenACC [11] and OpenMP4 [12]. Other proposals, such as the Hybrid Multi-core Parallel Programming model (HMPP) proposed by CAPS, hiCUDA [13], OpenMPC [14] and StarSs [15] follow the same line.

OpenACC today is considered among the most promising approaches to develop highperformance scientific applications [16]. In many ways its structure is similar to OpenMP (Open Multi-Processing) [17]: both frameworks are directive based, but while OpenMP is more prescriptive OpenACC is more descriptive. Indeed, with OpenACC the programmer only specifies that a certain loop should run in parallel on the accelerator and leaves the exact mapping to the compiler. This approach gives more freedom to the compiler and the associated runtime support, offering - at least in principle - larger scope for performance portability.

So far very few OpenACC implementations of LB codes have been described in literature: [18] focuses on accelerating via OpenACC a part of a large CFD application optimized for CPU; several other works describe CUDA [19] or OpenCL [20] implementations; also the scalability of OpenACC codes on GPU clusters has been rarely addressed [21]. This work describes the implementation of a state-of-the-art LB code fully written in OpenACC, including accurate performance measurements and an assessment of the actual portability improvements made possible by this programming style. This is an extended version of [22], that we have presented at the EuroPar 2015 conference. In the original paper, we focused on the design and optimization of a multi-GPU LB code, discussing performance trade-offs between a portable approach based on OpenACC and processor-specific languages such as CUDA. The present work includes additional material, discussing and analyzing issues related to the portability as well as the performance portability of our OpenACC codes. Our analysis is based on tests performed on several computing architectures, including multi-core CPUs and several different GPUs. In other words, the original paper provided an answer to the question of the performance price that one has to pay if one uses OpenACC instead of a processor-specific programming language, while this extended version also answers the question of how portable and performance-portable is just one architecture-oblivious OpenACC code across a fairly large set of different architectures.

Very recently Blair et al. have described an implementation of a MPI Lattice Boltzmann code with OpenACC [23]; however portability of code and performances across different architectures have not been analyzed; to the best of our knowledge, this paper is the first work discussing these issues for OpenACC.

This paper is structured as follows: Sect. 2 gives a short overview of LB methods and Sect. 3 a quick overview of the OpenACC programming framework; Sect. 4 describes in details our OpenACC implementation, and Sect. 5 analyzes performance results on GPUs in comparison with a CUDA implementation of the same code. Section 6 (added in the extended version) analyzes the portability of the same OpenACC code on different architectures: Intel E5-2630 v3 multicore CPUs, NVIDIA K80 and AMD S9150 GPUs. Finally, Sect.7highlights our conclusions.

\section{LATTICE BOLTZMANN MODELS}

Lattice Boltzmann methods (LB) are widely used in computational fluid dynamics, to describe flows in two and three dimensions. LB methods [24] are discrete in position and momentum spaces; they are based on the synthetic dynamics of populations sitting at the sites of a discrete lattice. At each time step, populations hop from lattice-site to lattice-site and then incoming populations collide among one another, that is, they mix and their values change accordingly.

Over the years, many different LB models have been developed, handling flows in 2 and 3 dimensions with different degrees of accuracy [25]. LB models in $n$ dimensions with $y$ populations are labeled as DnQy; in this paper, we consider a state-of-the-art $D 2 Q 37$ model that correctly reproduces the thermo-hydrodynamical equations of motion of a fluid in two dimensions and 

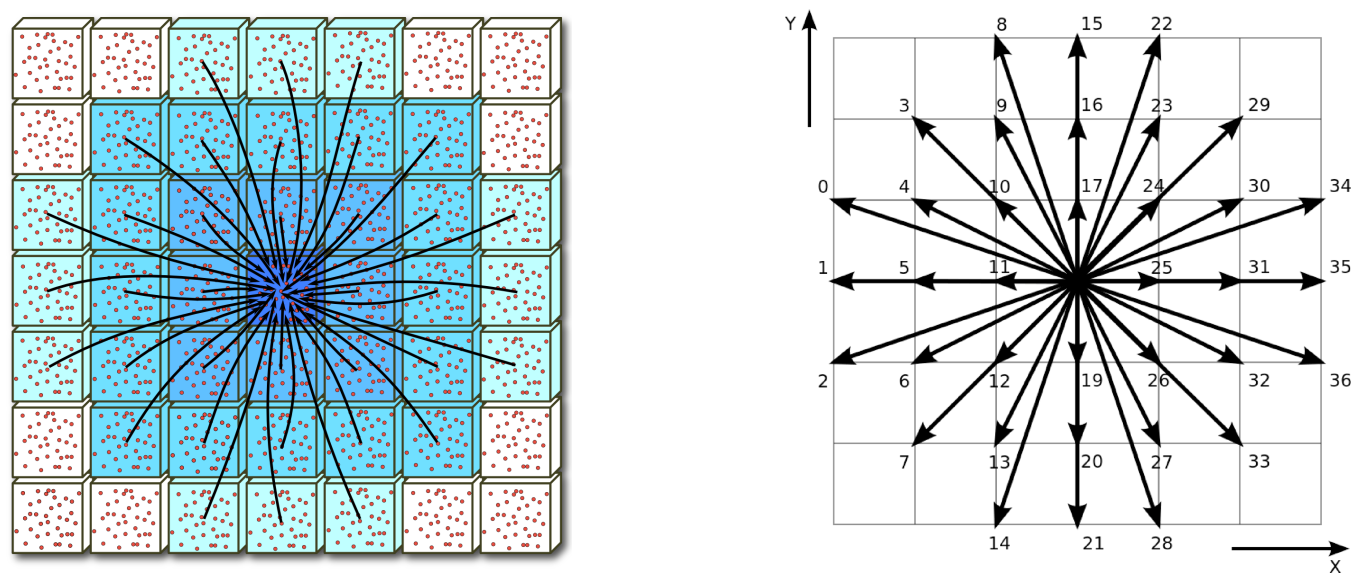

Figure 1. Left: LB populations in the D2Q37 model, hopping to nearby sites during the propagate phase. Right: populations $f_{l}$ are identified by an arbitrary label; for each $l$ population data is stored contiguously in memory.

automatically enforces the equation of state of a perfect gas $(p=\rho T)$ [26, 27]; this model has been extensively used for large scale simulations of convective turbulence (see e.g., [28, 29, 30]).

In our model, populations $\left(f_{l}(\boldsymbol{x}, t) l=1 \cdots 37\right)$ are defined at the sites of a discrete and regular 2D lattice; each $f_{l}(\boldsymbol{x}, t)$ has a given lattice velocity $\boldsymbol{c}_{l}$; populations evolve in (discrete) time according to the following equation (the BGK operator [31]):

$$
f_{l}(\boldsymbol{x}, t+\Delta t)=f_{l}\left(\boldsymbol{x}-\boldsymbol{c}_{l} \Delta t, t\right)-\frac{\Delta t}{\tau}\left(f_{l}\left(\boldsymbol{x}-\boldsymbol{c}_{l} \Delta t, t\right)-f_{l}^{(e q)}\right)
$$

Macroscopic quantities, density $\rho$, velocity $\boldsymbol{u}$ and temperature $T$ are defined in terms of the $f_{l}(x, t)$ and of the $c_{l} \mathrm{~s}$ ( $D$ is the number of space dimensions):

$$
\rho=\sum_{l} f_{l}, \quad \rho \boldsymbol{u}=\sum_{l} \boldsymbol{c}_{l} f_{l}, \quad D \rho T=\sum_{l}\left|\boldsymbol{c}_{l}-\boldsymbol{u}\right|^{2} f_{l}
$$

the equilibrium distributions $\left(f_{l}^{(e q)}\right)$ are known functions of these macroscopic quantities [24], and $\tau$ is a suitably chosen relaxation time. In words, (1) stipulates that populations drift from lattice site to lattice site according to the value of their velocities (propagation) and, on arrival at point $\boldsymbol{x}$, they interact among one another and their values change accordingly (collision). One can show that, in suitable limiting cases and after appropriate renormalizations are applied, the evolution of the macroscopic variables defined in (2) obeys the thermo-hydrodynamical equations of motion of the fluid.

From a computational point of view the physically very accurate LB scheme that we adopt is more complex than many simpler LB models. One specific optimization step of the algorithm applies a systematic projection onto a basis of Hermite polynomials to improve numerical stability; this translates into severe requirements in terms of required storage, memory bandwidth and floatingpoint throughput (at each time step, $\approx 7600$ double-precision floating point operations are performed per lattice point).

An LB code takes an initial assignment of the populations, in accordance with a given initial condition at $t=0$ on some spatial domain, and iterates (1) for all points in the domain and for as many time-steps as needed; boundary-conditions at the edges of the integration domain are enforced at each time-step by appropriately modifying population values at and close to the boundaries.

The LB approach offers a huge degree of easily identified parallelism. Indeed, (1) shows that the propagation step amounts to gathering the values of the fields $f_{l}$ from neighboring sites, corresponding to populations drifting towards $\boldsymbol{x}$ with velocity $\boldsymbol{c}_{l}$; the following step (collision) then performs all mathematical processing needed to compute the quantities in the r.h.s. of $(1)$, for each 


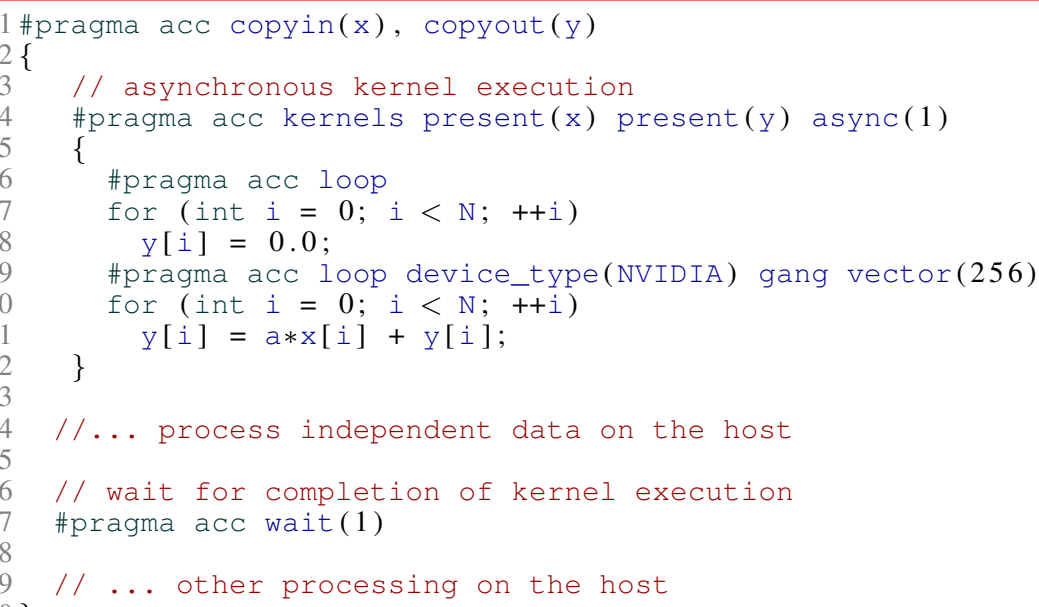

Figure 2. Sample OpenACC code computing a saxpy function on vectors $x$ and $y$. Directives mark the code region to run on the accelerator and instruct the compiler on how to generate code for the target device.

point in the grid. One sees immediately from (1), that both steps above are fully uncorrelated for different points of the grid, so they can be executed in parallel according to any schedule, as long as step 1 precedes step 2 for all lattice points.

In practice, an LB code executes a loop over time steps, and at each iterations applies three kernels: propagate, bc and collide.

- propagate moves populations across lattice sites according to the pattern of Figure 1 . collecting at each site all populations that will interact at the next phase (collide). In our model populations move up to three lattice sites per time step. Computer-wise, propagate moves blocks of memory locations allocated at sparse addresses, corresponding to populations of neighbor cells.

- bc executes after propagation and adjusts populations at the edges of the lattice, enforcing appropriate boundary conditions (e.g., constant temperature and zero velocity at the top and bottom edges of the lattice). For the left and right edges, we usually apply periodic boundary conditions. This is conveniently done by adding halo columns at the edges of the lattice, where we copy the rightmost and leftmost columns ( 3 in our case) of the lattice before starting the propagate step. After this is done, points close to the boundaries are processed as those in the bulk.

- collide performs all mathematical steps needed to compute the population values at each lattice site at the new time step, as per (1). Input data for this phase are the populations gathered by the previous propagate phase. This step is the most floating point intensive part of the code.

These three routines use essentially all the wall-clock time of a typical LB production run, as additional measurement routines, typically computing averages or correlations of physical observables, are invoked once every several hundreds or even thousands of time steps.

\section{OPENACC}

OpenACC is a programming standard for parallel computing aimed to facilitate code development on heterogeneous computing systems, simplifying the porting of existing codes and trying to achieve a significant level of performance portability. 


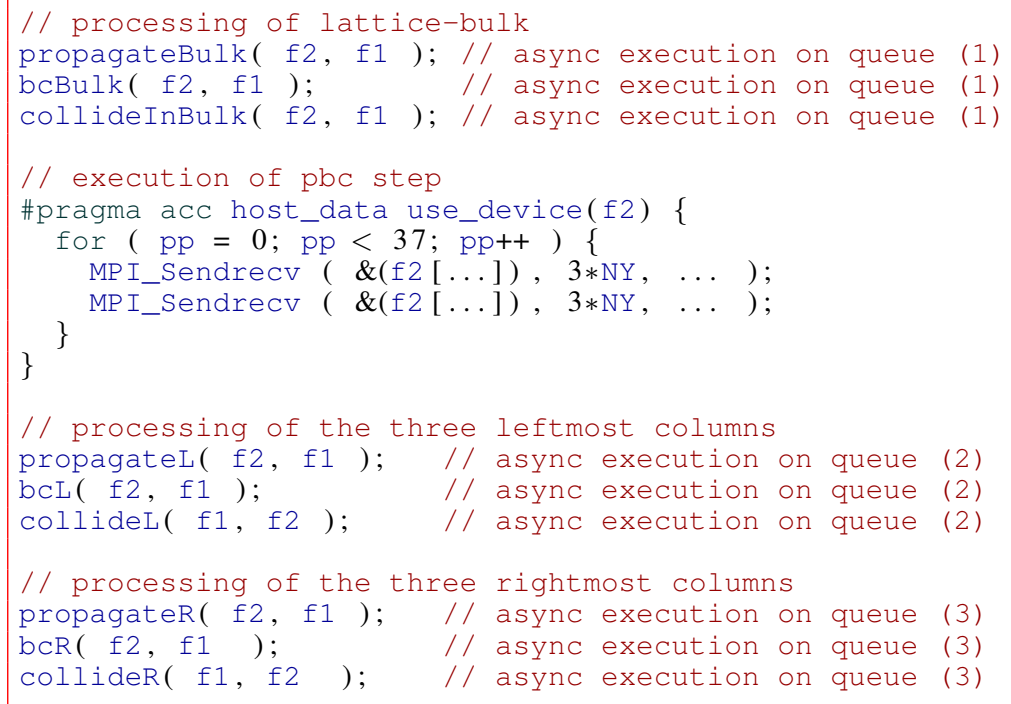

Figure 3. Scheduling of operations started by the host at each time step of the main program. Kernels processing the lattice bulk run asynchronously on the accelerator, and overlap with MPI communications executed by the host.

Its support for different architectures relies on compilers, and thanks to its generality the same code can be compiled and parallelized for different target architectures if the corresponding backend and run-time support are available. Recent versions of OpenACC implementations, such as the PGI release, version 15.10, can address as target architectures NVIDIA and AMD accelerators as well as commodity multicore $x 86$ CPUs [32].

OpenACC, like OpenCL, provides a widely applicable abstraction of parallel hardware, making it possible to run the same code across different architectures. Contrary to OpenCL, where specific functions (called kernels) have to be explicitly programmed to run in a parallel fashion (e.g. as GPU threads), OpenACC relies on developer-provided directives that help the compiler identify those parts of the source code that can be implemented as parallel functions. Following these directives the compiler generates one or more kernel functions - in the OpenCL sense - to be executed in parallel by many threads.

OpenACC is similar to the OpenMP (Open Multi-Processing) language in several ways [17]; both environments are directive based, but OpenACC targets accelerators in general, while so far OpenMP has been used to target mainly multi-core CPUs. The latest version of OpenMP [12] has recently introduced support for accelerators using a model very close to that of OpenACC. From a programmers perspective, OpenMP is more prescriptive in the sense that explicit mapping of workloads to compute-units, e.g. using distribute constructs, is required. In contrast to this OpenACC, being more descriptive, only requires the programmer to expose parallelism and let the compiler do the actual mapping to the compute-units. Since different hardware architectures require different mappings to perform efficiently, this approach makes OpenACC in principle more performance portable.

Existing $\mathrm{C} / \mathrm{C}++$ or Fortran code, developed and tested on traditional $\mathrm{CPU}$ architectures, can be annotated with OpenACC directives (e.g. parallel or kernels directives) to instruct the compiler to transform loop iterations into distinct threads, belonging to one or more functions to run on an accelerator. Figure 2 shows a simple example based on the saxpy operation from the Basic Linear Algebra Subprogram (BLAS) set.

Line 4 contains the pragma acc kernels directive which identifies the code to run on the accelerator; in this case the iterations of the two for-loops are parallelized and the execution of the function is offloaded at run-time from the host CPU to an attached accelerator device (e.g. a GPU). More directives are available, allowing a finer tuning of the application. As an example, the 


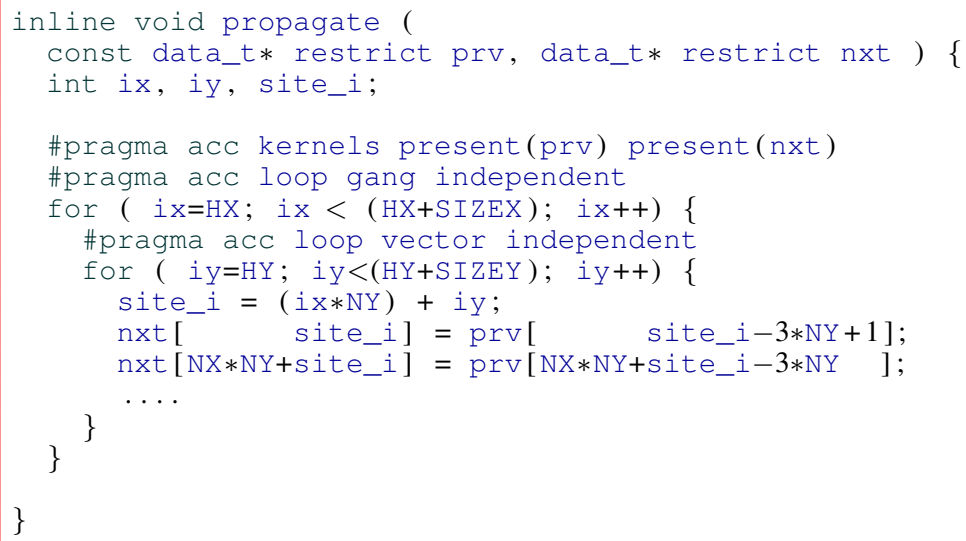

Figure 4. OpenACC pragmas in the body of the propagate ( ) function; pragmas before the loops instruct the compiler to generate corresponding accelerator kernels and to configure the grid of threads and blocks.

number of threads launched by each device function and their grouping can be fine tuned by the vector, worker and gang directives, in a similar fashion as setting the number of work-items and work-groups in OpenCL: in the example of Figure 2, line 9 sets vector length to 256. Since the gang and vector clauses encode a hardware specific tuning they follow a device_type clause, e.g., they only apply to the target specified as an argument of the device_type clause and allow hardware specific tuning without harming performance portability.

Data transfers between host and device memories are automatically generated, when needed. These automatically generated data movements are often overly cautious and thus can be optimized by the programmer with data directives. For example, in the code shown in Figure 2 the clause $\operatorname{copyin}(x)$ (at line 1) copies the array of the host CPU pointed by $x$ onto the accelerator memory before entering the following code region; while copyout( $y$ ) allocates an empty buffer before entering the region and copies it back to the host memory after leaving. Introducing this so called data region avoids multiple unnecessary copies:

1. a copy of $y$ from host memory to the accelerator memory before the loop in line 7 and the reverse copy after the loop.

2. a copy of $y$ from host memory to the accelerator memory before the loop in line 10

3. a copy of $x$ from the accelerator memory to host memory after the loop in line 10

The last OpenACC feature used in the example is the asynchronous clause async (at line 4) which instructs the compiler to generate asynchronous data transfers or kernel executions and allows overlapping of independent data transfers, kernels and CPU work. A directive corresponding to the async clause is provided by the OpenACC API (\#pragma acc wait at line 17) which allows to wait for completion. For more details on OpenACC features and functions see [11].

\section{OPENACC IMPLEMENTATION AND OPTIMIZATION OF THE D2Q37 MODEL}

This section describes in details the strategies that we have adopted to write an OpenACC version of our LB code suitable for compilation and execution on NVIDIA GPUs. In a later section, we will then focus on portability issues, as we experiment with this code on different HPC architectures and measure the corresponding performances.

One of our initial goals was to have a massively parallel program, able to run on a large number of GPUs. From the point of view of data organization, we adopt a very simple domain decomposition, splitting our 2-D physical lattice of size $L_{x}^{t o t} \times L_{y}$ on $N$ accelerators along the $X$ dimension; GPUs 


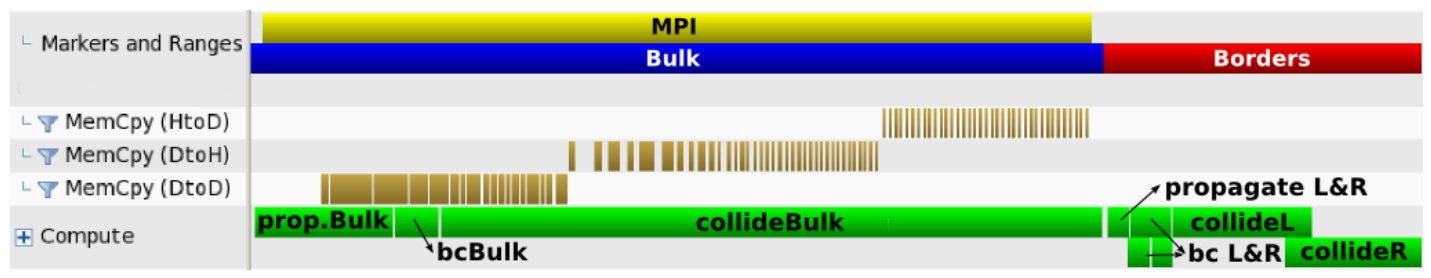

Figure 5. Profiling of one time step. In this example, pbc (yellow line marked as "MPI") and the kernels processing the bulk of the lattice (blue line marked as "Bulk") fully overlap.

are connected in a ring-scheme, each hosting a sub-lattice of $L_{x}^{t o t} / N \times L_{y}=L_{x} \times L_{y}$ points. We use MPI for the overall control of node-parallelism, starting one MPI rank for each GPU, so GPUto-GPU transfers are transparently handled by the MPI library; once this is done, we use OpenACC to annotate the code executed by each MPI rank.

On each MPI-rank the physical lattice is surrounded by halo columns and rows: for a physical sub-lattice of size $L_{x} \times L_{y}$, we allocate $N X \times N Y$ points, with $N X=H_{x}+L_{x}+H_{x}$ and $N Y=$ $H_{y}+L_{y}+H_{y}$. With this splitting, halo-columns are allocated at successive memory locations, so we do not need to gather halo data on contiguous buffers before communication.

Data is stored in memory in the Structure-of-Array (SoA) scheme, where arrays of all populations are stored one after the other. This helps exploit data-parallelism and enables data-coalescing when accessing data needed by work-items executing in parallel.

The lattice is copied on the accelerator memory at the beginning of the loop over time-steps, and then all three kernels of the algorithm - propagate, bc and collide - run in sequence on the accelerator for as many time-steps as needed. Merging in one step execution of propagate and collide is a common optimization in LBM codes. However, in our case this would require a more complex organization of the code to process separately lattice-sites that have no dependencies with bc kernel that runs before collide but after propagate. For this reason our implementation runs the two kernels in two separate steps; this is also useful for benchmark purposes since these kernels have different computing requirements being - in our LB model - the first memory-bound and the latter strongly compute-bound

The execution of these kernels starts after an update of the left- and right-halos is performed: we copy population data coming from the three adjoining physical columns of the neighbor nodes in the ring to the left and right halos. This is done by an MPI node-to-node communication step that we call periodic boundary condition ( $\mathrm{pbc}$ ). Once this is done, all remaining steps are local to each MPI-rank so they run in parallel.

As lattice data is stored in the SoA format, pbc exchanges 37 buffers, each of 3 columns, with its left and right neighbors. It executes a loop over the 37 populations and each iteration performs two MPI send-receive operations, respectively for the left and the right halo (see Figure 3). On GPUs, we exploit CUDA-aware MPI features, available in the OpenMPI library, and use data pointers referencing GPU-memory buffers as source and destination, making the code more compact and readable. In OpenACC this is controlled by the \#pragma acc host_data use_device (p) clause, that maps a GPU memory pointer $\mathrm{p}$ into host space, so it can be used as an argument of the MPI send and receive functions. Also, communications between GPUs are optimized in the library and implemented according to physical location of buffers and the capabilities of the devices involved, also enabling GPUDirect peer-to-peer and RDMA features.

Coming now to the main kernels of the algorithm, Figure 4 shows the code of the propagate function. For each lattice site we update the values of the populations, copying from the prv array onto the nxt array. The body of propagate is annotated with several OpenACC directives telling the compiler how to organize the kernel on the accelerator. \#pragma acc kernels present (prv) present (nxt) tells the compiler to run the following instructions on the accelerator; it also carries the information that the prv and nxt arrays are already available on the accelerator memory, so no host-accelerator data transfer is needed; \#pragma acc loop gang independent states that each iteration of the following loop (over the X-dimension) can be run 
by different gangs or block of threads; \#pragma acc loop vector independent tells the compiler that iterations of the loop over Y-dimension can likewise be run as independent vectors of threads. Using these directives the compiler structures the thread-blocks and block-grids of the accelerator computation in such a way that: one thread is associated to and processes one lattice-site; each thread-block processes a group of lattice sites lying along the Y-direction, and several blocks process sites along the $\mathrm{X}$-direction. This allows to expose all available parallelism.

We split bc () in two kernels, processing the upper and lower boundaries. They run in parallel since there is no data dependencies among them. We have not further optimized this step because its computational cost is small compared to the other phases of the code.

The collide () kernel sweeps all lattice sites and computes the collisional function. The code has two outer loops over the two dimensions of the lattice, and several inner loops to compute temporary values. We have annotated the outer loops as we did for propagate (), making each thread to process one lattice site. Inner loops are computed serially by the thread associated to each site.

Performance wise, $\mathrm{pbc}()$ is the most critical step of the multi-GPU code, since it involves node-to-node communications that can badly affect performance and scaling. We organize the code so node-to-node communications are (fully or partially) overlapped with the execution of other segments of the code. Generally speaking, propagate, bc and collide must execute one after the other, and they cannot start before pbc has completed. One easily sees however that this dependency does not apply to all sites of the lattice outside the three leftmost and rightmost border columns (we call this region the bulk of the lattice). The obvious conclusion is that processing of the bulk can proceed in parallel with the execution of pbc, while the sites on the three leftmost and rightmost columns are processed only after pbc has completed.

OpenACC abstracts concurrent execution using queues: function definitions flagged by the \#pragma acc async(n) directive enqueue the corresponding kernels asynchronously on queue $\mathrm{n}$, leaving the host free to perform other tasks concurrently. In our case, this happens for propagateBulk, bcBulk and collideBulk, which start on queue 1 (see Figure 3), while the host concurrently executes the MPI transfers of pbc. After communications complete, the host starts three more kernels on two different queues ( 2 and 3 ) to process the right and left borders, so they execute in parallel if sufficient resources on the accelerator are available. This structure allows to overlap pbc with all other steps of the code, most importantly with collideBulk, which is the most time consuming kernel, giving more opportunities to hide communication overheads when running on a large number of nodes.

Figure 5 shows the profiling of one time step on one GPU on a lattice of $1080 \times 2048$ points split across 24 GPUs. MPI communications started by pbc are internal (MemCopy DtoD), moving data between GPUs on the same host, or external (MemCopy DtoH and HtoD) moving data between GPUs on different hosts. The actual scheduling is as expected: both types of GPU-to-GPU communications fully overlap with propagate, bc and collide on the bulk.

\section{GPU RESULTS}

We start our performance analysis on NVIDIA GPUs, comparing our OpenACC code with an implementation of the same algorithm written in CUDA [33, 19] and optimized for Fermi and Kepler architectures. In other words, we compare with a low-level programming approach which gives programmers more freedom in mapping codes and data on GPU architectures, and then more optimization options.

Table II summarizes performance figures of codes on a reference lattice of $1920 \times 2048$ sites run on two NVIDIA systems, the K40 and K80 boards. These accelerator cards are powered by respectively the GK110B and GK210 processors based on the latest Kepler GPU family. The K40 has a peak memory bandwidth of $288 \mathrm{~GB} / \mathrm{s}$, and a peak floating-point performance of 1430 GFLOPs; this can increase up to 1660 GFLOPs boosting the GPU clock frequency to $875 \mathrm{MHz}$. The NVIDIA K80 is a dual-GPU system. Each GPU features a peak memory bandwidth of $240 \mathrm{~GB} / \mathrm{s}$, 
Table I. Performance comparison of OpenACC code with CUDA version running on NVIDIA Tesla K40 and K80 GPU accelerator cards; all codes run on a lattice size of $1920 \times 2048$ points. All quantities are defined in the text. The last two rows show the "wall-clock" execution time and the corresponding MLUPS (Millions Lattice UPdate per Second) for the full code.

\begin{tabular}{lrrrrrr}
\hline & \multicolumn{2}{c}{ Tesla K40 } & & \multicolumn{2}{c}{ Tesla K80 } \\
\cline { 2 - 3 } \cline { 6 - 7 } Code Version & CUDA & OACC & & CUDA & OACC \\
\hline$T_{\text {Prop }}[\mathrm{msec}]$ & 13.78 & 13.91 & & 7.60 & 7.51 \\
$\mathrm{~GB} / \mathrm{s}$ & 169 & 167 & & 306 & 310 \\
$\mathcal{E}_{p}$ & $59 \%$ & $58 \%$ & & $64 \%$ & $65 \%$ \\
\hline$T_{\mathrm{Bc}}[\mathrm{msec}]$ & 4.42 & 2.76 & & 1.11 & 0.71 \\
\hline$T_{\text {Collide }}[\mathrm{msec}]$ & 39.86 & 78.65 & & 16.80 & 36.39 \\
MLUPS & 99 & 50 & & 234 & 108 \\
$\mathcal{E}_{c}$ & $45 \%$ & $23 \%$ & & $52 \%$ & $24 \%$ \\
\hline$T_{\mathrm{WC}} /$ iter $[\mathrm{msec}]$ & 58.07 & 96.57 & & 26.84 & 44.61 \\
MLUPS & 68 & 41 & & 147 & 88 \\
\hline
\end{tabular}

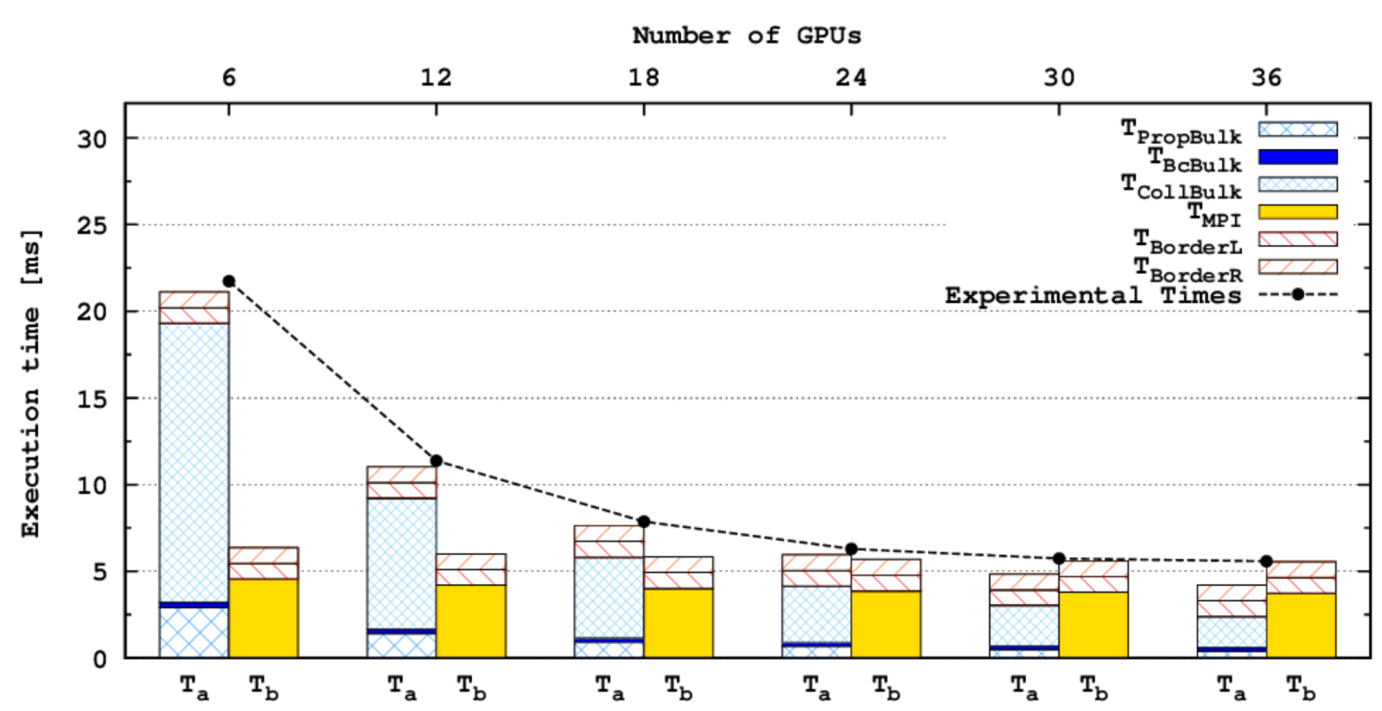

Figure 6. $T_{a}$ and $T_{b}$ for the time model defined in the text on a lattice of $1080 \times 5736$ points as a function of the number of GPUs. The black points are the execution times of the code with all asynchronous steps enabled.

and a peak double-precision floating-point performance of 935 GFLOPs; this can be increased up to 1455 GFLOPs, again boosting the GPU clock to $875 \mathrm{MHz}$.

We have used the PGI compiler version 14.10 for our test on the K40 and version 15.10 for running on the K80; while for CUDA we have used the NVIDIA compiler version 6.5 for the K40 and version 7.5 for the K80. Using more recent version of the compilers for the K40 does not changes the results. The codes executed on the K80 system runs two MPI ranks, each using one GPU of the same accelerator card.

The first line of Table \refers to the execution of propagate kernel. We show the execution time, the effective bandwidth, and the efficiency $\mathcal{E}_{p}$ computed w.r.t. the peak memory bandwidth of each system; the table then lists execution times of the bc function, showing that this routine has limited (albeit non negligible) impact on performance. For the collide kernel, we show the execution time and the efficiency $\mathcal{E}_{c}$ as a fraction of peak performance. Efficiency is computed using as number of double-precision operations for each lattice-site either the number measured by 


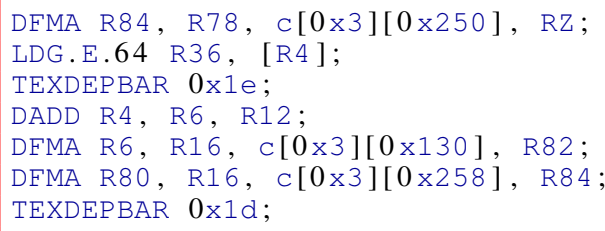

Figure 7. Part of SASS assembly of the CUDA collide kernel showing the use of constant cache values (operands $c[\ldots][\ldots]$ ) addressed directly by instructions.

the profiler through the hardware counters available on the processor or the number of floating-point instructions of the corresponding assembly code. Finally, the last two lines at bottom show the wallclock execution time (WcT) and the corresponding Millions Lattice UPdate per Second (MLUPS) - counting the number of sites handled per second - of the full production-ready code.

For propagate, which is strongly memory bound, the CUDA and OpenACC versions run at $\approx 60 \%$ of peak. For the collide kernel, which is the most computationally intensive part of the application, the OpenACC code has an efficiency of $\approx 25 \%$ on each system, while the CUDA version doubles this figure, running at $45 \%$ of peak on the $\mathrm{K} 40$ and $52 \%$ on the K80 thanks to the higher number of available registers.

Our analysis of the performance gap between OpenACC and CUDA codes for the collide kernel shows that a crucial role is played by the different way in which the coefficients of the Hermite polynomial expansion are stored. Our code uses 18 double-precision Hermite coefficients for each population, and the associated memory footprint to store them is $2368(=18 \times 37 \times 8)$ Bytes. Coefficients are initialized at run-time by the host and used as constant values by the kernels running on the GPU. CUDA allows explicit control on the allocation of data onto the various memory structures inside the GPU; in our case, the $64 \mathrm{~KB}$ low-latency constant cache of each GPU processor-core (SMX) is large enough to fit all coefficients, so they are copied there once before starting execution of the main loop. Data items stored in constant cache can be directly addressed by assembly instructions and do not require load operations onto registers (see figure 77). As a consequence, a larger number of general registers is then available to the compiler to fully unroll all inner loops of the collide routine. This allows to cache the accesses to the populations of a site onto registers improving performance significantly. Note also that data stored on the constantmemory is available to all threads, so no data replication on general registers is needed, and the performance impact is relevant in spite of the relatively small number of coefficients.

At a variance with the CUDA case, the OpenACC compiler can not identify the Hermite coefficients as constant values as they are initialized at run-time. The consequences of this are that: (a) they are loaded on the global memory, and accesses to them are handled as less efficient regular memory accesses; (b) registers are required to stage these coefficients, and for this reason inner loops can not be unrolled and code runs approximately $2 \mathrm{X}$ slower w.r.t. the CUDA version. Unrolling the inner-loops of collide by hand causes significant register-spilling (5432 bytes spill stores, 13368 bytes spill loads) harming the performance by approximately a factor 10X w.r.t the CUDA version. As a check of this analysis, we have verified that a CUDA version that does not use the constant-memory and does not unroll inner loops matches the performance of the OpenACC code version.

Drawing a temporary conclusion looking at the overall MLUPS delivered of the full code on each system, we have that performances of OpenACC code are $\approx 40 \%$ lower with respect to the CUDA code.

We now discuss in details the scaling behavior of our parallel implementation as we run it on an increasing number of GPUs. We model the execution time of the whole program as $T \approx$ $\max \left\{T_{a}, T_{b}\right\}$, with $T_{a}$ and $T_{b}$ defined as:

$$
T_{a}=T_{\text {bulk }}+T_{\text {borderL }}+T_{\text {borderR }}, \quad T_{b}=T_{\mathrm{MPI}}+T_{\text {borderL }}+T_{\text {borderR }}
$$




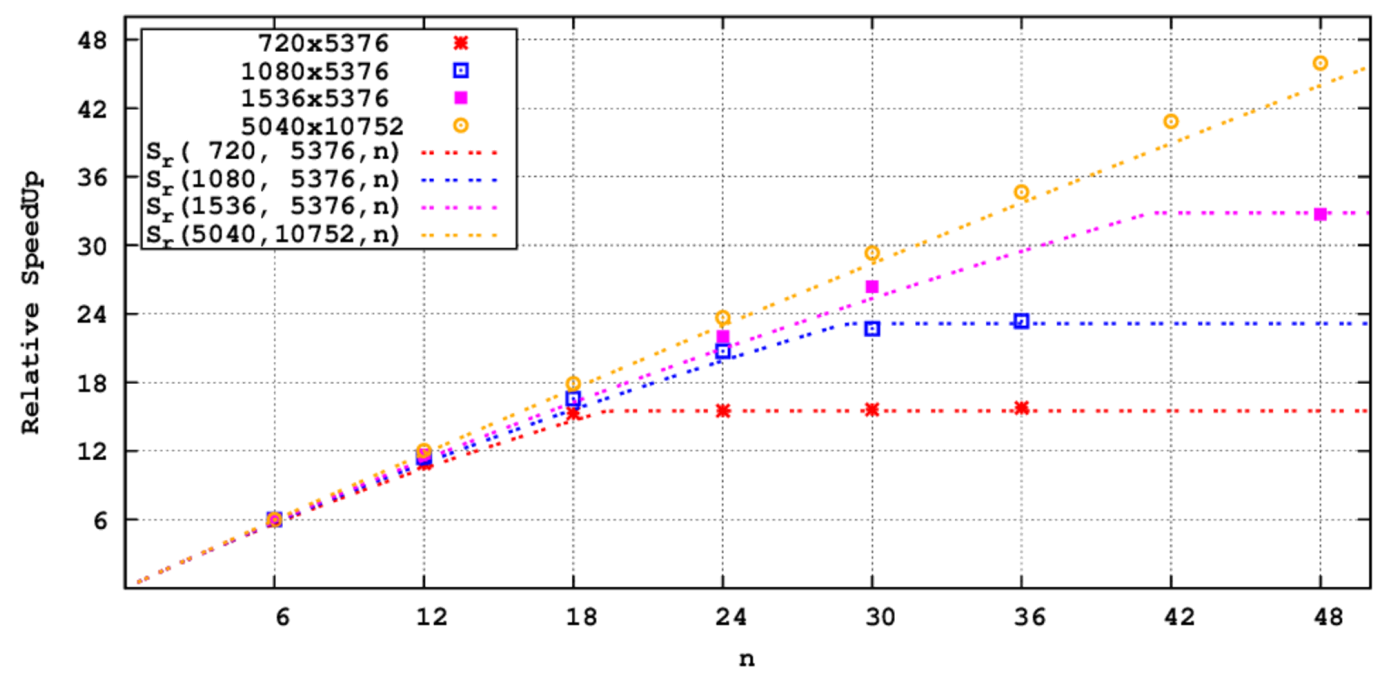

Figure 8. Strong scaling behavior of the OpenACC code as a function of the number of GPUs ( $n$ ) for several lattice sizes. Points are experimental data and dashed lines are the predictions of our timing model.

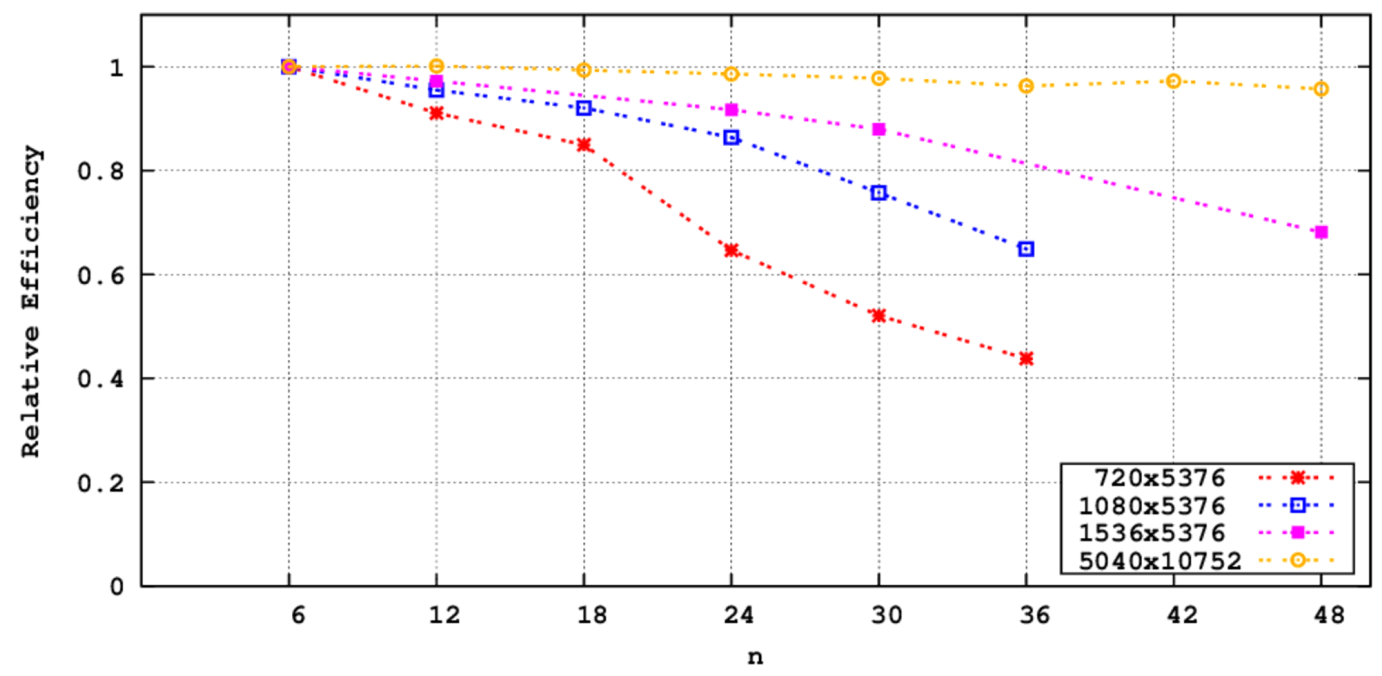

Figure 9. Parallel efficiency of the OpenACC code as a function of the number of GPUs ( $n$ ) for several lattice sizes.

and $T_{\text {bulk }}, T_{\text {borderL }}, T_{\text {borderR }}$ are respectively the sums of the execution times of propagate, bc and collide on the bulk, and on the left and right halos, while $T_{\text {MPI }}$ refers to MPI communications; we first profile the execution time of each kernel and MPI communication running them in sequence, i.e. without any overlap, and then we measure the execution time of the whole program with all asynchronous steps enabled.

This model is in good agreement with data measured on an Infiniband-interconnected cluster with 36 GPUs (6 GPUs on each node): Figure 6 shows our measured data for $T_{a}$ and $T_{b}$ on a lattice of $1080 \times 5736$ points. The histograms show the times taken by each section of the code when running serially while the black dots show the time taken by the asynchronous code. For this choice of the lattice size, we see that $T \approx T_{a}$ up to 24 GPUs as communications are fully hidden behind the execution of the program on the bulk; as long as this condition holds, the code enjoys full scalability. As we increase the number of GPUs $(\geq 30) T \approx T_{b}$, communications become the bottleneck and the scaling behavior necessarily degrades. 
Table II. Selected hardware features of the computing systems considered in this paper to assess portability of OpenACC codes. Clock freq. for Intel Xeon E5-2680 v3 are for AVX code.

\begin{tabular}{lrrcr}
\hline & Intel Xeon & NVIDIA K80 & AMD S9150 \\
\hline processor codename & E5-2630 v3 & \multicolumn{2}{c}{ GK210 } & Hawaii XT \\
\#physical-cores & 8 & 13 & x 2 & 44 \\
\#logical-cores & 16 & 2496 & x 2 & 2816 \\
nominal clock Freq. (GHz) & 2.1 & 0.562 & & 0.900 \\
Nominal GFLOPS (DP) & 268.625 & 935 & x 2 & 2530 \\
Max Boosted clock Freq. (GHz) & 2.6 & 0.875 & & - \\
Boosted GFLOPS (DP) & 331.56 & 1455 & x 2 & - \\
Max Memory (GB) & 768 & 12 & x 2 & 16 \\
Mem Bandwidth (GB/s) & 59 & 240 & x 2 & 320 \\
ECC & YES & YES & & YES \\
\hline
\end{tabular}

We further characterize the execution time assuming, to first approximation, that bulk processing is proportional to $\left(L_{x} \times L_{y}\right)$, boundary conditions scale as $L_{x}$, and communication and border processing scales as $L_{y}$; so, on $n$ GPUs

$$
T\left(L_{x}, L_{y}, n\right)=\max \left\{\alpha \frac{L_{x}}{n} L_{y}+\beta \frac{L_{x}}{n}, \quad \gamma L_{y}\right\}+\delta L_{y}
$$

We extract the parameters $(\alpha, \beta, \gamma$ and $\delta)$ from the profiling data of Figure 6 , and define the function

$$
S_{r}\left(L_{x}, L_{y}, n\right)=\frac{T\left(L_{x}, L_{y}, 1\right)}{T\left(L_{x}, L_{y}, n\right)}
$$

to predict the relative speedup for any number of GPUs and any lattice size. Figure 8 shows the (strong) scaling behavior of our code for several lattice sizes relevant for physics simulations; dots are measured values and dashed lines are plots of $S_{r}()$ for different values of $L_{x}$ and $L_{y}$. Values of $S_{r}()$ are in good agreement with experimental data, and predict the number of GPUs at which scaling violations start to become important. For large lattices $(5040 \times 10752)$ the code has an excellent scaling behavior up to 48 GPUs, slightly underestimated by our model as constants are calibrated on smaller lattices so they are more sensitive to overheads. Figure 9 shows the corresponding parallel efficiency of our code. For large lattices it remains close to one, while for smaller lattice using the largest number of GPUs is in the range 40-60\%.

\section{OPENACC PORTABILITY}

In this section we analyze the portability of the OpenACC version described in the previous sections across several architecture taking into account also portability of performances.

In this work, we consider three different target computing systems supported by the PGI OpenACC compiler: an $x 86$ commodity multi-core processor, and two accelerators, an NVIDIA K80 and an AMD FirePro S9150 GPU. Together with the Intel Xeon-Phi this set of architectures is currently the most used in HPC systems. The PGI compiler so far does not support Xeon-Phi so we cannot include this accelerator in our comparison; for performance details of our LB code on Xeon-Phi using other frameworks see [9, 37].

The Intel Xeon processor is an 8-core E5-2630 v3 CPU based on the recent Haswell microarchitecture. For this processors the base clock frequency is $2.1 \mathrm{GHz}$ for applications using the AVX vector-unit, like the LB code analyzed here; enabling turbo-mode and using all 8 cores available the frequency can boosts up to $2.6 \mathrm{GHz}$ for these kind of applications. This CPU has then a peak memory bandwidth of $59 \mathrm{~GB} / \mathrm{s}$, and a a peak double-precision floating-point performance of $\approx 330$ GFLOPs; the PGI compiler supports generating multi threaded code from OpenACC for this family 


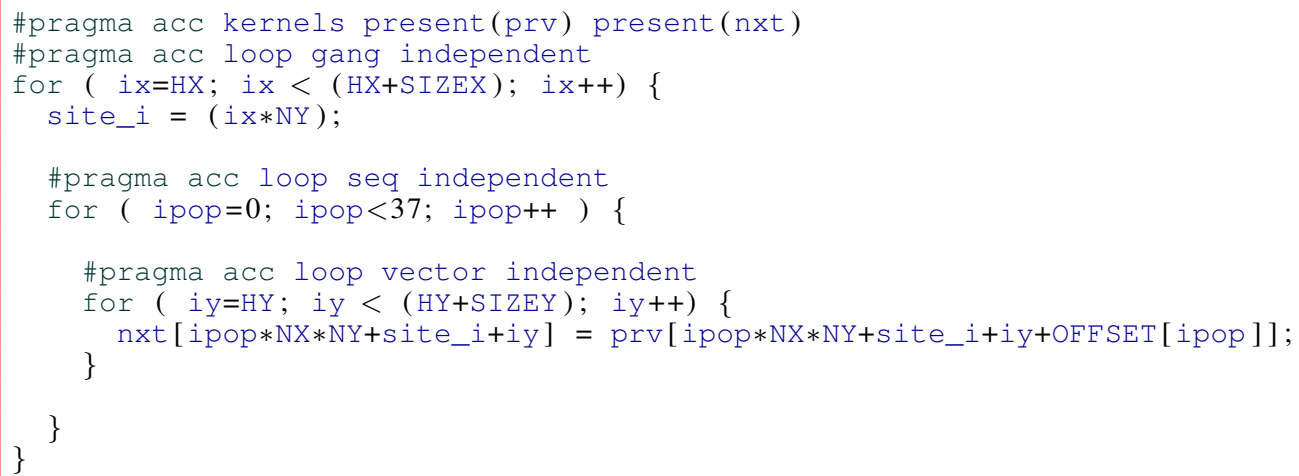

Figure 10. Code snapshot of the propagate kernel, version v2, that improves performances on $x 86$ CPUs.

of processors since version 15.9. The NVIDIA K80 system (whose details have been introduced in Sect. 5] is a dual-GPU system, and for our benchmarks we have used only one of the two GK210 GPUs, whose features are very similar to those of the NVIDIA K40 GPU considered in previous sections, so our results can be directly compared with those shown in Table I. Finally, the AMD S9150 is a GPU accelerator with a memory bandwidth of $320 \mathrm{~GB} / \mathrm{s}$ and up to 2620 GFLOPs doubleprecision floating point performance. Table II summarizes several hardware parameters useful for our analysis.

The target architecture for the compilation is specified by appropriate options (e.g., -ta=radeon and -ta=nvidia for AMD and NVDIA GPUs respectively and -ta=multicore for $x 86$ multicore CPUs).

In the $x 86$ case, the parallelization performed by the compiler is similar to that implied by the omp parallel OpenMP directive. Gangs of OpenACC loops and regions are run on different physical-cores, or virtual-cores if hyper-threading is enabled. The compiler uses all available cores on the processor unless a different number is specified by the gang clause or through the ACC_NUM_CORES environment variables. The directive acC vector is considered as a hint indicating that the compiler can vectorize the loop, but the compiler uses its own analysis to determine if vectorization can be applied, and generate SIMD code. A multicore CPU is treated as a shared-memory accelerator, so data clauses (like copy, copyin, copyout, create) are ignored and no data copies are executed.

In all cases, it is useful to keep an eye on the strategies taken by the compiler using compiler options -Minfo or - Minfo=accel that enable feedback messages, giving some details on the parallel and/or vectorized code generated.

We have run and benchmarked the propagate and collide kernels on the three systems described above. From the point of view of code portability results are really good as exactly the same C-code (the one described in previous sections), annotated with the same OpenACC pragmas, immediately runs on all three architectures.

Table III. Benchmark results for the propagate and collide kernels on three different processor architectures. Both kernels are compiled using the PGI compiler, version 15.10.

\begin{tabular}{lccc}
\hline & E5-2630 v3 & GK210 & Hawaii XT \\
\hline propagate v1 [GB/s] & 10 & 155 & 216 \\
propagate v2 [GB/s] & 32 & 145 & 223 \\
\hline collide v1 [MLUPS] & 8 & 55 & 53 \\
collide v2 [MLUPS] & 12 & 6 & 5 \\
\hline
\end{tabular}




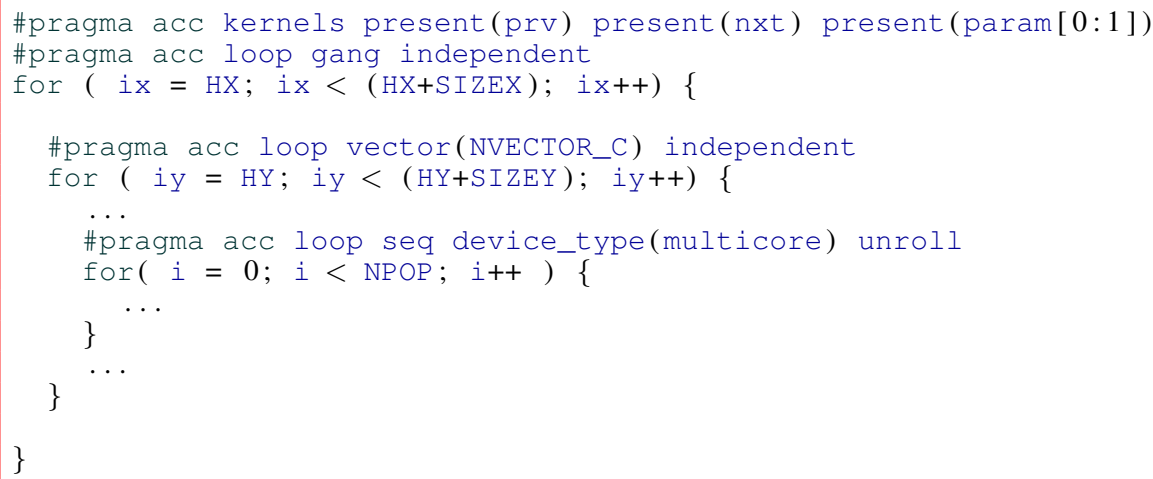

Figure 11. Example usage of the proposed unroll clause.

However results are not fully satisfying from the point of view of performance portability, see table III, codes version v1. For instance, performances on the AMD GPU increases for propagate in line with the higher available memory bandwidth but performance for collide remains roughly the same for both GPUs, in spite of the significantly higher peak value of the AMD accelerator. On the $x 86 \mathrm{CPU}$, results are even more worrying, with a larger drop in performance for both kernels.

Trying to improve performances, we experimented with a different organization of the propagate kernel: while in the original code (version v1, see again Figure 4) for each lattice site we move all 37 populations associated to it, the new version (called v2, see Figure 10) processes lattice-sites by columns: for each column and for each population index we handle in sequence all sites of the column.

Performances of the two kernels run on all target architectures are shown on table Table III. Comparing version v2 with v1 we see that on CPUs it gives better performances increasing the effective memory bandwidth by a factor $3 \mathrm{X}$. The measured value is $32 \mathrm{~GB} / \mathrm{s}$ corresponding to $54 \%$ of the raw peak. On NVIDIA and AMD GPUs both versions give approximately (within 10\%) the same result.

We have also experimented with a new version of the collide kernel (version v2) in which we unroll most internals loops by hand. Also in this case version v2 gives better results on $x 86$ CPUs increasing performance by a factor $1.5 \mathrm{X}$ w.r.t. version v1. On GPUs however version v2 is much slower. Unrolling most internal loops increases the number of registers needed per thread. This causes register spilling and the resulting local memory overhead causes the slowdown of v2 on GPUs. As shown in Figure 11 a unroll clause for the loop directive combined with the device_type clause would allow to maintain the same code for multicore CPUs and GPUs.

Table IV] collects our final comparison results; it summarizes the best results that we have measured on all target architectures using the OpenACC PGI compiler and the performances of the same computational kernels coded with other programming approaches, closer to each specific architecture. In details, for $x 86$ CPUs we show results of two multi-thread codes compiled with the Intel compiler version 15: one uses intrinsics functions [34, 35] to exploit vectorization, while the latter [36] uses OpenMP directives; both use OpenMP to handle multicore parallelization. For NVIDIA GPUs we consider the CUDA code, while for AMD GPUs we have used GCC and OpenCL [20, 37].

Table IV provides several metrics for a comparative assessment of code portability: for computeintensive kernels one may consider the ratio of delivered floating-point performance w.r.t peak performance on different machines; for data-intensive application a better parameter may be the ratio of measured memory bandwidth w.r.t peak; finally one may want to compare the performance of the OpenACC code with the corresponding performance of the same code written in an architecture-specific programming language.

Having this in mind, several comments are in order: 
Table IV. Performance comparison of various programming frameworks on various processors. MLUPS stands for Mega-Lattice Updates per Second; the performance in GFLOPs is obtained assuming that each lattice points uses 6500 floating-point operations; Total performance (Tot perf.) is evaluated on a program that invokes in sequence propagate and collide.

\begin{tabular}{lrrr|rr|rr}
\hline & \multicolumn{3}{c|}{ E5-2630 v3 } & \multicolumn{2}{c|}{ GK210 } & \multicolumn{2}{c}{ Hawaii XT } \\
\hline compiler & ICC 15 & ICC 15 & PGI 15.10 & NVCC 7.5 & PGI 15.10 & GCC & PGI 15.10 \\
model & Intrinsics & OMP & OACC & CUDA & OACC & OCL & OACC \\
\hline propagate perf. [GB/s] & 38 & 32 & 32 & 154 & 155 & 232 & 216 \\
$\mathcal{E}_{p}$ & $65 \%$ & $54 \%$ & $54 \%$ & $64 \%$ & $65 \%$ & $73 \%$ & $70 \%$ \\
\hline collide perf. [MLUPS] & 14 & 11 & 12 & 117 & 55 & 76 & 54 \\
collide perf. [GFLOPs] & 92 & 71 & 78 & 760 & 356 & 494 & 351 \\
$\mathcal{E}_{c}$ & $28 \%$ & $22 \%$ & $24 \%$ & $52 \%$ & $24 \%$ & $19 \%$ & $14 \%$ \\
\hline Tot perf. [MLUPS] & 11.5 & 9.2 & 9.8 & 80.7 & 45.6 & 63.7 & 47.0 \\
\hline
\end{tabular}

- on the $x 86 \mathrm{CPU}$, the three versions of the code have remarkably the same level of performances for both kernels. All runs are using all 8 CPU-cores available and have turbo-mode enabled. Using intrinsics is slightly more efficient, but the more "user-friendly" programming frameworks are almost as efficient (within $\approx 10 \%$ ). For the collide kernel the main bottleneck for performances is the memory latency in accessing Hermite coefficients and population values needed to compute this kernel for each lattice site.

- On the NVIDIA GPU, the OpenACC code gives the same performance of CUDA for the propagate kernel. Results are not the same for the collide kernel where we measure a drop of $2 \mathrm{X}$ compared w.r.t the CUDA version. Reasons for this behaviour have been analyzed in the Sect. 5

- On the AMD GPU, we have almost equally efficient propagate versions with the two available programming environments; but the performance of the collide kernel is lower for OpenCL and OpenACC. In this case, we have not analyzed the performance bottleneck in depth; for this reason it is possible that performance could be optimized further.

Trying to provide a global assessment of the performance portability offered by the PGI OpenACC compiler, we first remark that it is able to produce very efficient code for the propagate kernel exploiting a large fraction of the memory bandwidth offered by all processors; this is substantiated by the reported figures for $\mathcal{E}_{p}$ (i.e., by the sustained memory bandwidth w.r.t. peak memory bandwidth).

For the compute intensive collide kernels, OpenACC comes at the cost of a non negligible performance gap; considering in this case $\mathcal{E}_{c}$ (measuring the actual floating point performance), we see a performance drop of $2 X$.

As a global assessment, the last line of Table $\mathrm{IV}$ shows an effective performance metric for the whole code assuming to execute propagate and collide in sequence; this gives a lower-bound of performances of the program since the two routines can often (actually depends on routines to be run between the two) be merged in one single step. Keeping this point in mind, our figures show that OpenACC is indeed able to support code portability at the price of a performance drop lower than $50 \%$, and with reasonable expectations of further improvements in the future. As an unexpected aside, we also show that the PGI compiler is remarkably efficient for multi-core $x 86$ CPUs.

Finally, we have successfully run the same OpenACC code used for the GPU cluster also on a cluster of Intel Xeon E5-2630 v3 CPUs. Each node of the cluster hosts two eight-core CPUs, and the nodes are interconnected via Infiniband QDR network. The code runs two MPI ranks per node, and each MPI process "offloads" execution of the kernels on the 8-cores of the CPU on which it is running. Figure 12 shows the strong scalability behaviour achieved on 16 nodes of the cluster corresponding to $32 \mathrm{CPUs}$ and 256 cores, using several lattice sizes with different aspect ratios. Figure 13 shows the corresponding parallel efficiency. Contrary to the case of a GPU cluster, in this case we do not have a real overlap between communication and computation. Both are concurrently 


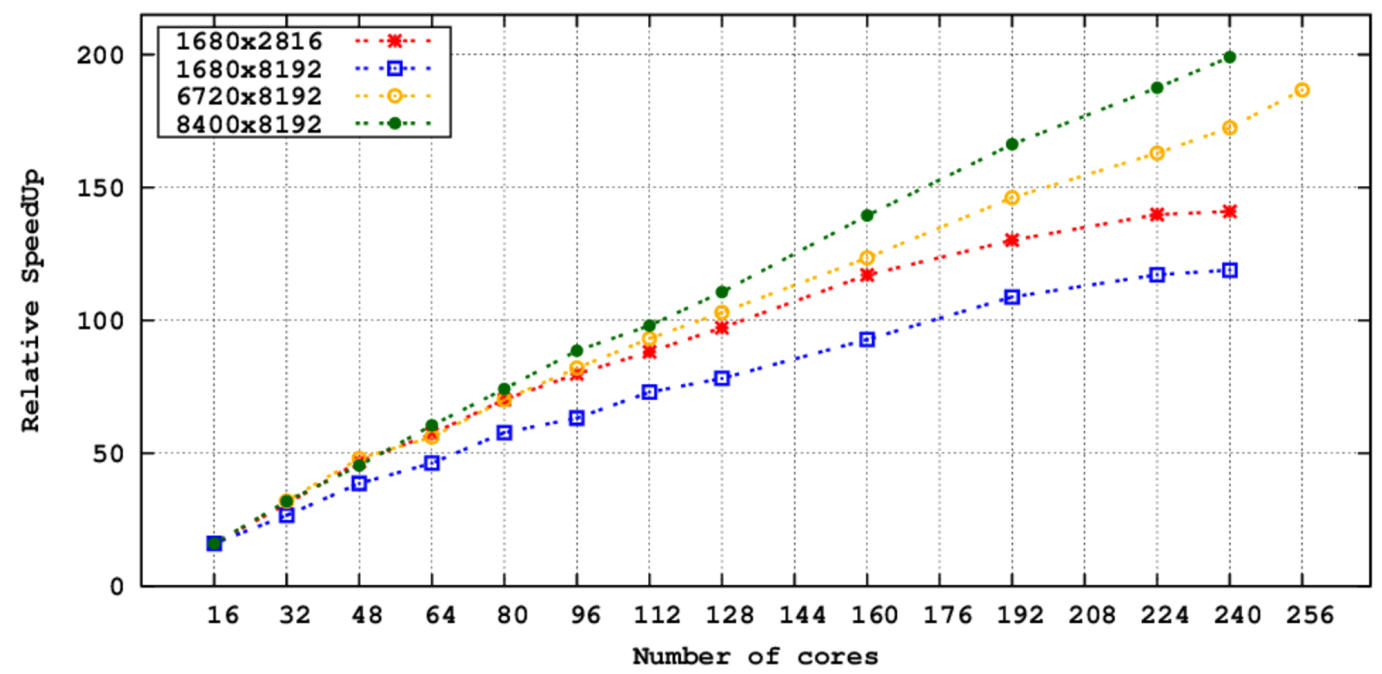

Figure 12. Strong scaling behaviour of our OpenACC code as function of the number of CPU-cores. The code run on a X86 CPU cluster interconnected via Infiniband QDR network.

managed by the cores of CPUs, and this limits scaling behaviour. For the lattices we have tried, using the largest number (240-256) of CPU-cores the relative speedup is in the range 120-200, and the parallel efficiency results in the range $50-80 \%$.

\section{CONCLUSIONS}

In conclusion, we have successfully ported, tested and benchmarked a complete multi-node Lattice Boltzmann code using OpenACC, and characterized its performances through an accurate timing model.

Our experience with OpenACC is very positive from the point of view of code portability and programmability. The effort to port existing codes to OpenACC is reasonably limited and easy

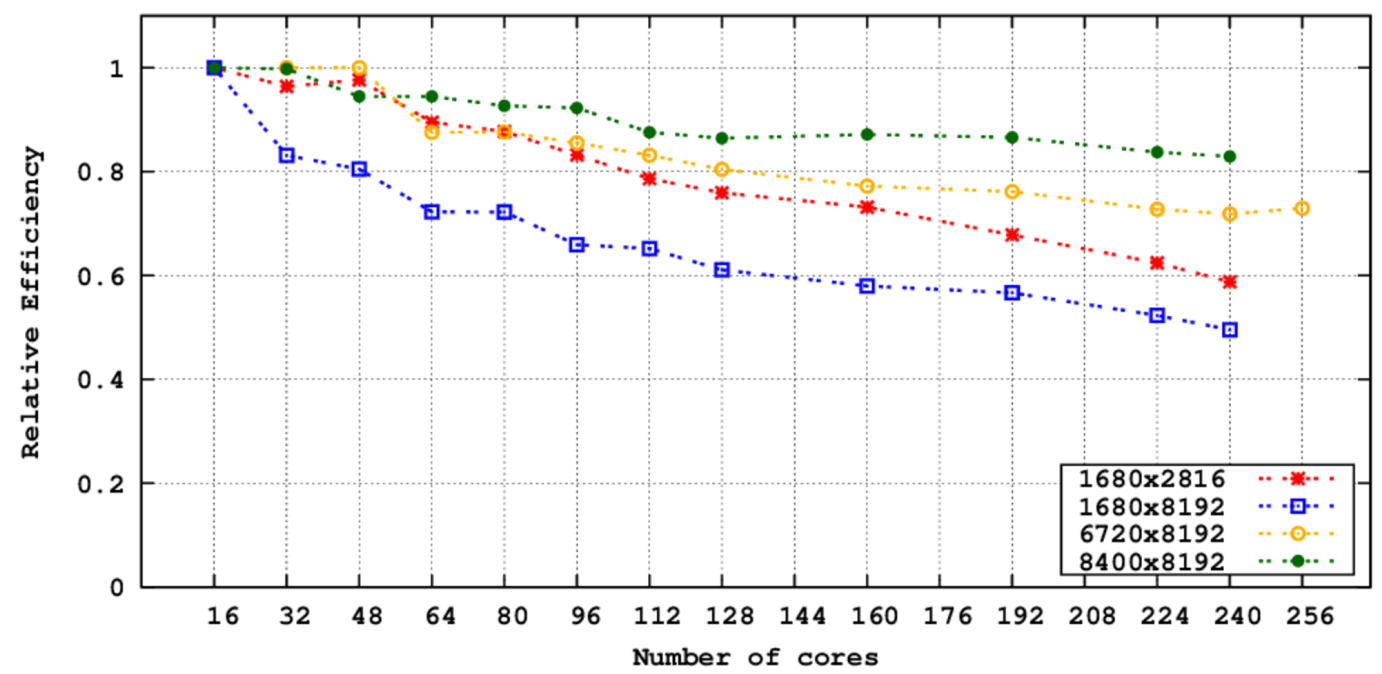

Figure 13. Parallel efficiency of our OpenACC code as function of the number of CPU-cores. The code run on a X86 CPU cluster interconnected via Infiniband QDR network. 
to handle; we started from an existing $\mathrm{C}$ version and marked through directives regions of code to offload and run on accelerators, instructing the compiler to identify and exploit available parallelism. However, we have to underline that some major changes in the global structure of codes and in data organization cannot be handled automatically by compilers and the overall organization must be (at least partially) aware of the target architectures. For example, in our case it is crucial to organize data as Structure of Arrays to allow to coalesce performance-critical memory accesses, and exploit vectorization.

Concerning code portability across different target architectures, our experience shows that the PGI compiler easily allows to target different processors widely used today in most HPC systems. As we have shown in Sect 6 the latest version of the compiler is able to target both $x 86$ multi-core CPUs and NVIDIA and AMD GPUs. We consider this a major result enabling users to quickly and easily benchmark a single-code on a wide range of target processors and decide which hardware better fits the computing requirements of applications. However also in this case we have to say that for optimal performances some changes in the organization of the codes may be required. In our case, for example, on $x 86$ multi-core CPUs we improved the performance of the propagate kernel by a factor $3 \mathrm{X}$ changing the order of processing of the lattice sites. On the other hand, applying this changes on codes written in a high level language annotated by OpenACC is much simpler than doing the same on codes written in OpenCL or heavily using intrinsic functions. This of course improves productivity of programmers and encourages experimentation with different codes.

Concerning performance results, one is ready to accept that using a high level programming model trades better programmability with computing efficiency, and a performance drop $\leq 20 \%$ may be considered a satisfactory result. Our experiments show that actual performances drop can be larger, approximately around a $2 X$ factor in our case, with overall performances that may be $\approx 50 \%$ of what is made possible by using more processor-specific programming methodologies. In many cases, as explained and discussed in Sect. 5 5 we understand the reasons behind this gap and have good reasons to believe that future versions of the compiler may introduce supports to narrow this performance gap. For example, in our case support for the constant cache available on NVIDIA GPUs would be useful. As an interim step, one may work around, e.g. exploiting the interoperability between OpenACC and CUDA for NVIDIA GPUS, to foster the high productivity of OpenACC and still get full performance by using CUDA for the most performance critical kernels. Similar approaches also apply to CPUs and AMD GPUs.

We believe that our analysis provides important feedback to help users understand the capabilities of the OpenACC approach as well as several hints to improve the performance of OpenACC codes.

In the short term future, we plan to test our OpenACC LB codes on yet more processors architectures, e.g. Intel Xeon-Phi, as soon as OpenACC support becomes available.

\section{ACKNOWLEDGMENTS.}

This work was done in the framework of the COKA, COSA and Suma projects of INFN. We thank INFN-Pisa (Pisa, Italy), the NVIDIA Jülich Application Lab (Jülich Supercomputer Center, Jülich, Germany) and the CINECA (Bologna, Italy) for allowing us to use their computing systems. AG has been supported by the European Union's Horizon 2020 research and innovation programme under the Marie Sklodowska-Curie grant agreement No 642069.

\section{REFERENCES}

1. Pohl, T., et al.: Performance evaluation of parallel large-scale lattice boltzmann applications on three supercomputing architectures. In: Proc. Conf. on Supercomputing (2004) doi:10.1109/SC.2004.37

2. Belletti, F. et al.: Multiphase lattice boltzmann on the cell broadband engine. Nuovo Cimento della Società Italiana di Fisica C 32(2) 53-56 (2009) doi:10.1393/ncc/i2009-10379-6

3. Biferale, L., et al.: Lattice boltzmann fluid-dynamics on the QPACE supercomputer. Proc. Comp. Science 1 10751082 (2010) doi:10.1016/j.procs.2010.04.119

4. Pivanti, M., et al.: An optimized lattice boltzmann code for BlueGene/Q. Lecture Notes in Computer Science 8385 385-394 (2014) doi:10.1007/978-3-642-55195-6_36 
5. Sano, K., et al.: FPGA-based streaming computation for lattice boltzmann method. In: Field-Programmable Technology 233-236 (2007) doi:10.1109/FPT.2007.4439254

6. Biferale, L., et al.: Optimization of multi-phase compressible lattice boltzmann codes on massively parallel multicore systems. Proc. Comp. Science 4 994-1003 (2011) doi:10.1016/j.procs.2011.04.105

7. Biferale, L., et al.: A multi-gpu implementation of a D2Q37 lattice boltzmann code. Lecture Notes in Computer Science 7203 640-650 (2012) doi:10.1007/978-3-642-31464-3_65

8. Bailey, P., et al.: Accelerating lattice Boltzmann fluid flow simulations using graphics processors. In: Parallel Processing 550-557 (2009) doi:10.1109/ICPP.2009.38

9. Crimi, G., et al.: Early Experience on Porting and Running a Lattice Boltzmann Code on the Xeon-phi CoProcessor. Proc. Comp. Science 18 551-560 (2013) doi:10.1016/j.procs.2013.05.219

10. Wienke, S., et al.: OpenACC-first experiences with real-world applications. In: Euro-Par Parallel Processing Springer 859-870 (2012) doi:10.1007/978-3-642-32820-6_85

11. OpenACC directives for accelerators. http: //www. openacc-standard.org/ last visited 2016-04-19

12. OpenMP. http://www. openmp.org/mp-document s/OpenMP 4 .0.0.pdf last visited 2016-04-19

13. Han, T., Abdelrahman, T.: hiCUDA: High-Level GPGPU Programming. Par. and Distr. Systems, IEEE Trans. 22(1) 78-90 (2011) doi:10.1109/TPDS.2010.62

14. Lee, S., Eigenmann, R.: OpenMPC: Extended OpenMP Programming and Tuning for GPUs. Proc. of SC 1-11 (2010) doi:10.1109/SC.2010.36

15. Ayguadé, E., et al.: Extending OpenMP to Survive the Heterogeneous Multi-Core Era. Int. J. of Parallel Programming 38(5-6) 440-459 (2010) doi:10.1007/s10766-010-0135-4

16. Bonati, C., et al.: Development of Scientific Software for HPC Architectures Using Open ACC: The Case of LQCD. IEEE/ACM 1st International Workshop on Software Engineering for High Performance Computing in Science (2015) doi:10.1109/SE4HPCS.2015.9

17. Wienke, S., et al.: A pattern-based comparison of OpenACC and OpenMP for accelerator computing. Lecture Notes in Computer Science 8632 812-823 (2014) doi:10.1007/978-3-319-09873-9-68

18. Kraus, J., et al: Accelerating a C++ CFD code with OpenACC. Proc. of the First Workshop on Accelerator Programming Using Directives 47-54 (2014) doi:10.1109/WACCPD.2014.11

19. Kraus, J., at al.: Benchmarking GPUs with a parallel Lattice-Boltzmann code. Comp. Arch. and High Perf. Computing (SBAC-PAD) 160-167 (2013) doi:10.1109/SBAC-PAD.2013.37

20. Calore, E., et al.: A Portable OpenCL Lattice Boltzmann Code for Multi-and Many-core Processor Architectures. Proc. Comp. Science 29 40-49 (2014) doi:10.1016/j.procs.2014.05.004

21. Obrecht, C., et al.: Scalable lattice Boltzmann solvers for CUDA GPU clusters. Parallel Computing 39(6-7) 259270 (2013) doi:10.1016/j.parco.2013.04.001

22. Calore, E., et al.: Accelerating Lattice Boltzmann applications with OpenACC. Lecture Notes in Computer Science 9233 613-624 (2015) doi:10.1007/978-3-662-48096-0_47

23. Blair, S., et al: Accelerating an MPI Lattice Boltzmann Code Using OpenACC. Proc. of the Second Workshop on Accelerator Programming Using Directives 3:1-3:9 (2015) doi:10.1145/2832105.2832111

24. Succi, S.: The Lattice-Boltzmann Equation. Oxford university press (2001)

25. Aidun, C.K., Clausen, J.R.: Lattice-Boltzmann Method for Complex Flows. Annual Review of Fluid Mechanics 42(1) 439-472 (2010) doi:10.1146/annurev-fluid-121108-145519

26. Sbragaglia, M., et al.: Lattice Boltzmann method with self-consistent thermo-hydrodynamic equilibria. J. of Fluid Mechanics 628 299-309 (2009) doi:10.1017/S002211200900665X

27. Scagliarini, A., et al.: Lattice Boltzmann methods for thermal flows: Continuum limit and applications to compressible Rayleigh-Taylor systems. Physics of Fluids 22(5) 055101 (2010) doi:10.1063/1.3392774

28. Biferale, L., et al.: Second-order closure in stratified turbulence: Simulations and modeling of bulk and entrainment regions. Phys. Review E 84(1) 016305 (2011) doi:10.1103/PhysRevE.84.016305

29. Biferale, L., et al.: Reactive Rayleigh-Taylor systems: Front propagation and non-stationarity. EPL (Europhysics Letters) 94(5) 54004 (2011) doi:10.1209/0295-5075/94/54004

30. Ripesi, L., et al.: Evolution of a double-front Rayleigh-Taylor system using a graphics-processing-unit-based highresolution thermal lattice-Boltzmann model. Phys. Review E. 89(4) (2014) doi:10.1103/PhysRevE.89.043022

31. Bhatnagar P. L., Gross E. P., Krook M., A Model for Collision Processes in Gases. I. Small Amplitude Processes in Charged and Neutral One-Component Systems. Phys. Rev. 94 511-525 (1954) doi:10.1103/PhysRev.94.511

32. Performance Portability from GPUs to CPUs with OpenACC. http://devblogs.nvidia.com/ parallelforall/performance-portability-gpus-cpus-openacc/ last visited 2016-04-19

33. L. Biferale et al., An Optimized D2Q37 Lattice Boltzmann Code on GP-GPUs, Computers and Fluids 80 55-62 (2013) doi:10.1016/j.compfluid.2012.06.003

34. Mantovani, F. et al, Exploiting parallelism in many-core architectures: Lattice Boltzmann models as a test case. J. Phys. Conf. Series 454012015 (2013) doi:10.1088/1742-6596/454/1/012015

35. Mantovani, F., et al.: Performance issues on many-core processors: A D2Q37 Lattice Boltzmann scheme as a testcase. Computers \& Fluids 88 743-752 (2013) doi:10.1016/j.compfluid.2013.05.014

36. Calore, E., et al.: Experience on vectorizing Lattice Boltzmann kernels for multi- and many-core architectures. Lecture Notes in Computer Science 9573 53-62 (2016) doi:10.1007/978-3-319-32149-3_6

37. Calore, E., et al.: On portability, performance and scalability of an MPI OpenCL lattice boltzmann code. Lecture Notes in Computer Science 8806 438-449 (2014) doi:10.1007/978-3-319-14313-2_37 